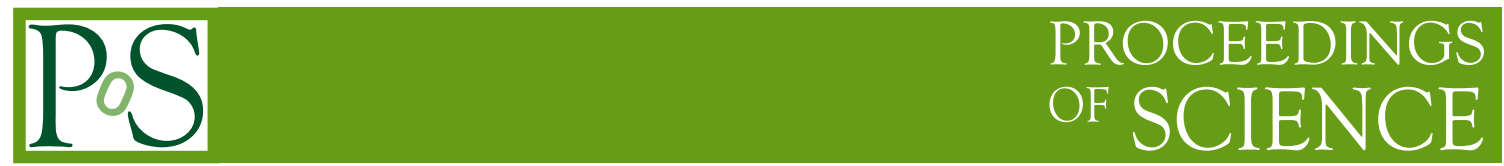

\title{
Introduction to Gravitational Microlensing
}

\section{Shude Mao*}

Jodrell Bank Centre for Astrophysics, University of Manchester, Manchester M13 9PL, UK

E-mail: shude.mao@manchester.ac.uk

The basic concepts of gravitational microlensing are introduced. We start with the lens equation, and then derive the image positions and magnifications. The statistical quantities of optical depth and event rate are then described. We finish with a summary and a list of challenges and open questions. A problem set is given for students to practice.

The Manchester Microlensing Conference: The 12th International Conference and ANGLES Microlensing Workshop

January 21-25 2008

Manchester, $U K$

*Speaker. 


\section{Introduction}

Gravitational microlensing (in the local group) refers to the temporal brightening of a background star due to intervening objects. Einstein (1936) first examined (micro)lensing by a single star, and concluded that "there is no great chance of observing this phenomenon." Some important works were performed in intervening years by [45] and [33], but the research topic was revitalised by Paczyński (1986) who proposed it as a method to detect compact dark matter objects in the Galactic halo.

The original goal is now out of favour, since we know with high precision that most of the dark matter must be non-baryonic, e.g. from observations of microwave background radiation and nucleosynthesis (at the time of his paper, this was, however, unclear). Nevertheless, gravitational microlensing has turned into a powerful technique with diverse applications in astrophysics, including the study of the structure of the Milky Way, stellar atmospheres and the detection of extrasolar planets and stellar-mass black hole candidates. The field has made enormous progress in the last two decades. There have been a number of reviews on this topic (e.g. [38, 35, 19, 51]), the most recent highlight was given in [25]. This article gives an introduction to microlensing, aimed at a level for a starting $\mathrm{PhD}$ student. Together with other talks in the workshop and proceedings ${ }^{1}$, one can gain a thorough feeling about the state-of-the-art research in this field (as of 2008).

The reference list given here is seriously incomplete (and biased). For more complete references and information about ongoing microlensing surveys, see the review papers mentioned above and the web site: http://mlens . net/ (built by Szymon Kozłowski, Subo Dong and Lukasz Wyrzykowski).

\section{What is gravitational microlensing?}

The light from a background source is deflected, distorted and (de)magnified by intervening objects along the line of sight. If the lens, source and observer are sufficiently well aligned, then strong gravitational lensing can occur. Depending on the lensing object, strong gravitational lensing can be divided into three areas: microlensing by stars, multiple-images by galaxies, and giant arcs and large-separation lenses by clusters of galaxies. For microlensing, the lensing object is a stellarmass compact object (e.g. normal stars, brown dwarfs or stellar remnants [white dwarfs, neutron stars and black holes]); the image splitting in this case is usually too small (of the order of milliarcsecond in the local group) to be resolved by ground-based telescopes, thus we can only observe the magnification change as a function of time.

The left panel in Fig. 1 illustrates the microlensing geometry. A stellar-mass lens moves across the line of sight towards a background star. As the lens moves closer to the line of sight, its gravitational focusing increases, and the background star becomes brighter. As the source moves away, the star falls back to its baseline brightness. If the motions of the lens, the observer and the source can be approximately taken as linear, then the light curve is symmetric. Since the lensing probability for microlensing in the local group is of the order of $10^{-6}$ (see section 5), the microlensing variability usually should not repeat. Since photons of different wavelengths follow the same propagation path (geodesics), the light curve (for a point source) should not depend on

\footnotetext{
1 available at http://pos.sissa.it/cgi-bin/reader/conf.cgi?confid=54
} 

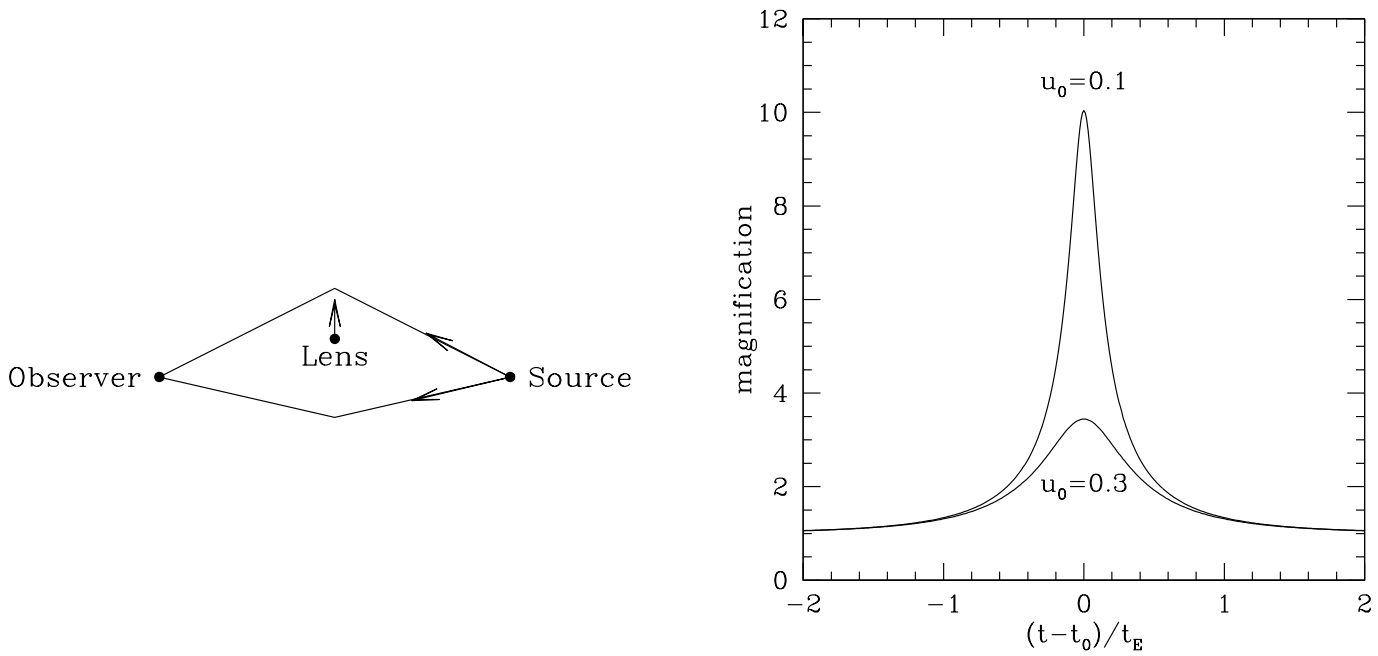

Figure 1: The left panel shows a side-on view of the geometry of microlensing where a lens moves across the line of sight towards a background source. The right panel shows two light curves corresponding to two dimensionless impact parameters, $u_{0}=0.1$ and 0.3 . The time on the horizontal axis is centred on the peak time $t_{0}$ and is normalised to the Einstein radius crossing time $t_{\mathrm{E}}$. The lower the value of $u_{0}$, the higher the peak magnification. For the definitions of $u_{0}$ and $t_{\mathrm{E}}$ see section 4.1.

the colour. The characteristic symmetric shape, non-repeatability, and achromaticity can be used as criteria to separate microlensing from other types of variable stars (exceptions to these rules will be discussed in section 4.2).

\section{Lens equation, image positions and magnifications}

To derive the characteristic light curve shape shown in the right panel of Fig. 1, we must look closely at the lens equation, and the resulting image positions and magnifications for a point source.

\subsection{Lens equation}

The lens equation is straightforward to derive. Figure 2 illustrates a side-on view of the lensing configuration. Simple geometry yields

$$
\vec{\eta}+D_{\mathrm{ds}} \hat{\hat{\alpha}}=\vec{\xi} \cdot \frac{D_{\mathrm{s}}}{D_{\mathrm{d}}}
$$

where $D_{\mathrm{d}}, D_{\mathrm{s}}$ and $D_{\mathrm{ds}}$ are the distance to the lens (deflector), distance to the source and distance between the lens (deflector) and the source, $\vec{\eta}$ is the source position (distance perpendicular to the line connecting the observer and the lens), $\vec{\xi}$ is the image position, and $\hat{\vec{\alpha}}$ is the deflection angle. For gravitational microlensing in the local group, $D_{\mathrm{ds}}=D_{\mathrm{s}}-D_{\mathrm{d}} \cdot{ }^{2}$ Mathematically, the lens equation provides a mapping between the source plane to the lens plane. The mapping is not necessarily one-to-one.

\footnotetext{
${ }^{2}$ For cosmological microlensing in an expanding universe, the distances should be taken as angular diameter distances, and in general $D_{\mathrm{ds}} \neq D_{\mathrm{s}}-D_{\mathrm{d}}$. See the review by Wambsganss in these proceedings on cosmological microlensing.
} 


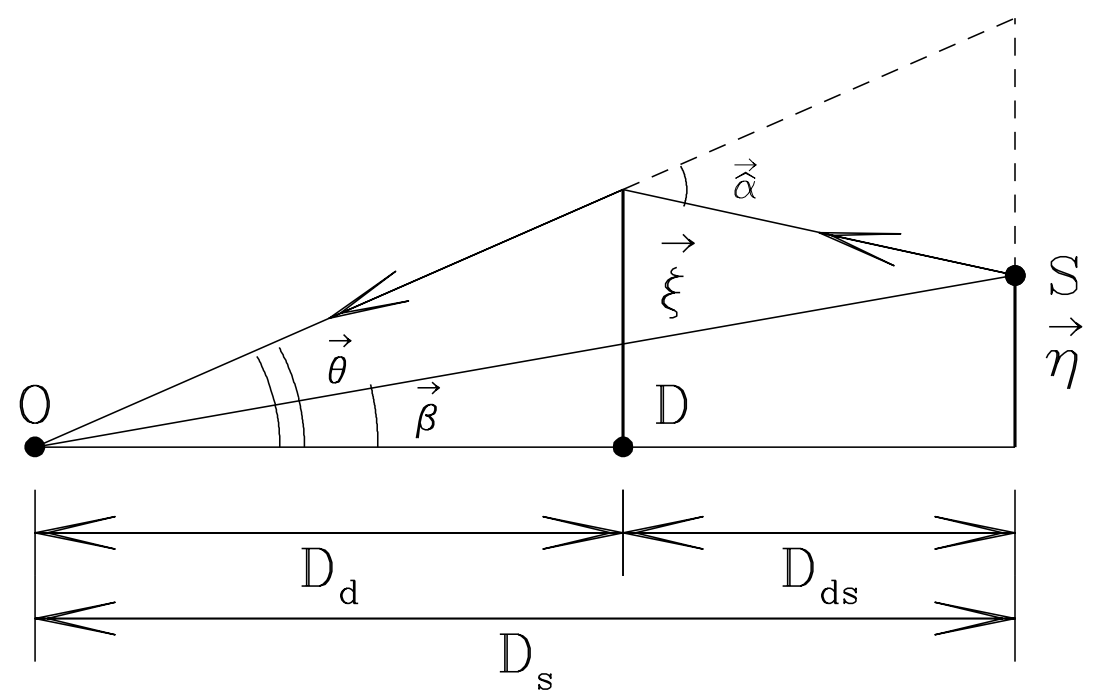

Figure 2: Illustration of various distances and angles in the lens equation (eqs. 3.1 and 3.2).

Dividing both sides of eq. (3.1) by $D_{\mathrm{s}}$, we obtain the lens equation in angles

$$
\vec{\beta}+\vec{\alpha}=\vec{\theta},
$$

where $\vec{\beta}=\vec{\eta} / D_{\mathrm{s}}, \vec{\theta}=\vec{\xi} / D_{\mathrm{d}}$, and $\vec{\alpha}=\hat{\vec{\alpha}} \times D_{\mathrm{ds}} / D_{\mathrm{s}}$ is the scaled deflection angle. These angles are illustrated in Fig. 2.

For an axis-symmetric mass distribution, due to symmetry, the source, observer and image positions must lie in the same plane, and so we can drop the vector sign, and obtain a scalar lens equation:

$$
\beta+\alpha=\theta
$$

\subsection{Image positions for a point lens}

For a point lens at the origin, the deflection angle is given by

$$
\hat{\vec{\alpha}}=\frac{4 G M}{c^{2}} \frac{1}{\xi^{2}} \vec{\xi}
$$

and the value of the scaled deflection angle is

$$
\alpha=\frac{D_{\mathrm{ds}}}{D_{\mathrm{s}}}|\hat{\hat{\alpha}}|=\frac{D_{\mathrm{ds}}}{D_{\mathrm{s}}} \frac{4 G M}{c^{2} D_{\mathrm{d}} \theta} \equiv \frac{\theta_{\mathrm{E}}^{2}}{\theta}, \quad \xi=D_{\mathrm{d}} \theta .
$$

where we have defined the angular Einstein radius as

$$
\theta_{\mathrm{E}}=\frac{r_{\mathrm{E}}}{D_{\mathrm{d}}} \approx 0.55 \mathrm{mas} \sqrt{\frac{1-D_{\mathrm{d}} / D_{\mathrm{s}}}{D_{\mathrm{d}} / D s}}\left(\frac{D_{\mathrm{s}}}{8 \mathrm{kpc}}\right)^{-1 / 2}\left(\frac{M}{0.3 M_{\odot}}\right)^{1 / 2} .
$$

The lens equation for a point lens in angles is therefore

$$
\beta+\frac{\theta_{\mathrm{E}}^{2}}{\theta}=\theta .
$$


We can further simply by normalising all the angles by $\theta_{\mathrm{E}}, r_{\mathrm{s}} \equiv \beta / \theta_{\mathrm{E}}, r \equiv \theta / \theta_{\mathrm{E}}$, the above equation becomes $^{3}$

$$
r_{\mathrm{s}}+\frac{1}{r}=r
$$

For the special case when the lens, source and observer are perfectly aligned $\left(r_{\mathrm{s}}=0\right)$, due to axissymmetry along the line of sight, the images form a ring ("Einstein" ring) with its angular size given by eq. (3.6).

For any other source position $r_{\mathrm{s}} \neq 0$, there are always two images, their positions are given by

$$
r_{ \pm}=\frac{r_{\mathrm{s}} \pm \sqrt{r_{\mathrm{s}}^{2}+4}}{2}
$$

The ' + ' image is outside the Einstein radius $\left(r_{+} \geq 1\right)$ on the same side of the source, while the '-' image is on the opposite side and inside the Einstein radius $\left(r_{-}<0\right.$ and $\left.\left|r_{-}\right|<1\right)$. The angular separation between the two images is

$$
\Delta \theta=\theta_{\mathrm{E}}\left(r_{+}-r_{-}\right)=\theta_{\mathrm{E}} \sqrt{4+r_{\mathrm{s}}^{2}} .
$$

The image separation is of the same order of the angular Einstein diameter when $r_{\mathrm{s}} \lesssim 1$, and thus will be in general too small to be observable given the typical seeing from the ground ( $\sim$ one arcsecond); we can only observe lensing effects through magnification. One exception may be the VLT interferometer (VLTI) which can potentially resolve the two images. This may be important for discovering stellar-mass black holes since they have larger image separations due to their larger masses than typical lenses with mass $\sim 0.3 M_{\odot}([13,42])$.

The physical size of the Einstein radius in the lens plane is given by

$$
r_{\mathrm{E}}=D_{\mathrm{d}} \theta_{\mathrm{E}}=\sqrt{\frac{4 G M}{c^{2}} \frac{D_{\mathrm{d}} D_{\mathrm{ds}}}{D_{\mathrm{s}}}} \approx 2.2 \mathrm{AU} \sqrt{4 \times \frac{D_{\mathrm{d}}}{D_{\mathrm{s}}}\left(1-\frac{D_{\mathrm{d}}}{D_{\mathrm{s}}}\right)}\left(\frac{D_{\mathrm{s}}}{8 \mathrm{kpc}}\right)^{1 / 2}\left(\frac{M}{0.3 M_{\odot}}\right)^{1 / 2} .
$$

So the size of the Einstein ring is roughly the scale of the solar system, which is a coincidence that helps the discovery of extrasolar planets around lenses.

\subsection{Image magnifications}

Since gravitational lensing conserves surface brightness, the magnification of an image is simply given by the ratio of the image area and source area. For a very small source, we can consider a thin source annulus with angle $\Delta \phi$ (see Fig. 3). For a point lens, this thin annulus will be mapped into two annuli, one inside the Einstein ring and one outside.

The area of the source annulus is given by the product of the radial width and the tangential length $d r_{\mathrm{s}} \times r_{\mathrm{s}} \Delta \phi$. Similarly, each image area is $d r \times r \Delta \phi$, and the magnification is given by ${ }^{4}$

$$
\mu=\frac{d r \times r \Delta \phi}{d r_{\mathrm{s}} \times r_{\mathrm{s}} \Delta \phi}=\frac{r}{r_{\mathrm{s}}} \frac{d r}{d r_{\mathrm{s}}}
$$

For the two images given in eq. (3.12), one finds

$$
\mu_{+}=\frac{\left(r_{\mathrm{s}}+\sqrt{r_{\mathrm{s}}^{2}+4}\right)^{2}}{4 r_{\mathrm{s}} \sqrt{r_{\mathrm{s}}^{2}+4}}, \mu_{-}=-\frac{\left(r_{\mathrm{s}}-\sqrt{r_{\mathrm{s}}^{2}+4}\right)^{2}}{4 r_{\mathrm{s}} \sqrt{r_{\mathrm{s}}^{2}+4}} .
$$

\footnotetext{
${ }^{3} r_{\mathrm{s}}$ is not to be confused with the size of the star, which we denote as $r_{\star}$.

${ }^{4}$ This is a special case of the determinant of the Jacobian in the lens mapping, see section 4.3.
} 
The magnification of the ' + ' image is positive, while the ' - ' image is negative. The former image is said to have positive parity while the latter negative ${ }^{5}$. The total magnification is given by

$$
\mu=\left|\mu_{+}\right|+\left|\mu_{-}\right|=\frac{r_{\mathrm{s}}^{2}+2}{r_{\mathrm{s}} \sqrt{r_{\mathrm{s}}^{2}+4}},
$$

and the difference is identical to unity

$$
\left|\mu_{+}\right|-\left|\mu_{-}\right|=1 .
$$

We make some remarks about the total magnification and image separations:

1. When $r_{\mathrm{s}}=1, \mu=3 / \sqrt{5} \approx 1.342$, corresponding to about 0.319 magnitude. Such a difference is easily observable ${ }^{6}$, and so the area occupied by the Einstein ring is usually taken as the lensing "cross-section."

2. When $r_{\mathrm{s}} \rightarrow \infty,\left|\mu_{+} / \mu_{-}\right| \rightarrow r_{\mathrm{s}}{ }^{4}, \mu \rightarrow 1+2 r_{\mathrm{s}}{ }^{-4}$. The angular image separation is given by $\Delta \theta=\left(r_{\mathrm{s}}+2 r_{\mathrm{s}}^{-1}\right) \theta_{\mathrm{E}}$.

3. High magnification events occur when $r_{\mathrm{s}} \rightarrow 0$. The asymptotic behaviours are $\mu \rightarrow r_{\mathrm{s}}{ }^{-1}(1+$ $\left.3 r_{\mathrm{s}}^{2} / 8\right), \Delta \theta \rightarrow\left(2+r_{\mathrm{s}}^{2} / 4\right) \theta_{\mathrm{E}}$, and $d r / d r_{\mathrm{s}} \rightarrow 1 / 2$. The last relation implies that, at high magnification, the image is compressed by a factor of 2 in the radial direction (see Fig. 3).

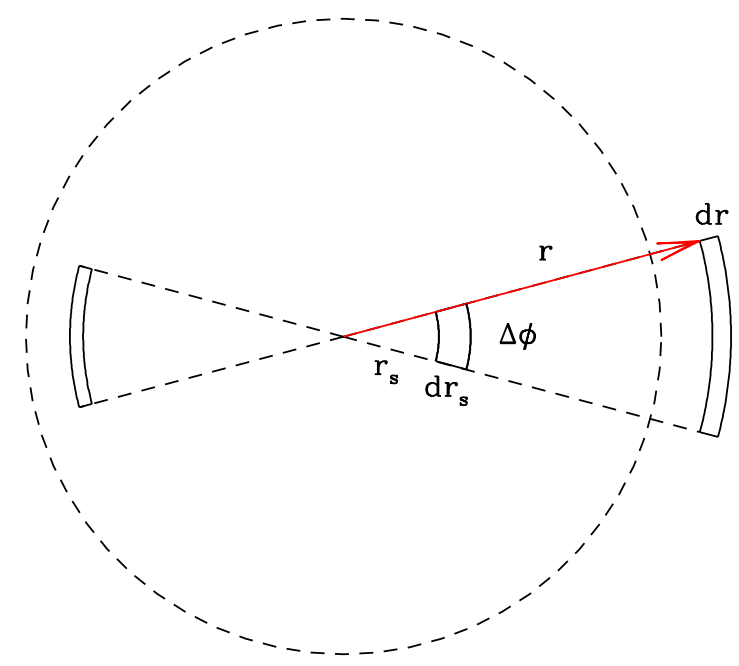

Figure 3: Images of a thin annulus from $r_{\mathrm{s}}$ to $r_{\mathrm{s}}+d r_{\mathrm{s}}$ by a point lens on the plane of the sky. The dashed line is the Einstein ring. $\Delta \phi$ is the angle subtended by the thin annulus.

\section{Light curve and microlensing degeneracy}

Given a source trajectory, we can easily describe the standard light curve with a few parameters which suffers from the microlensing degeneracy.

\footnotetext{
${ }^{5}$ Let us imagine two arrows for the thin annulus (see Fig. 3), one in the radial direction and one in the tangential direction respectively. For the negative parity image, the corresponding tangential arrow for the image is reversed with respect to that in the source, while in the radial direction the arrow directions remain the same for the source and image. For the positive parity image, the directions of the arrows are the same for the image and the source.

${ }^{6}$ For bright stars, the accuracy of photometry can reach a few milli-magnitudes.
} 


\subsection{Standard light curve}

For convenience, we put the lens at the origin, and let the source move across the line of sight along the $x$-axis (see Fig. 4). The impact parameter in units of the Einstein radius is labelled as $u_{0}$. For convenience, we define the Einstein radius crossing time (or 'timescale') as

$$
t_{\mathrm{E}}=\frac{r_{\mathrm{E}}}{v_{\mathrm{t}}}=\frac{\theta_{\mathrm{E}}}{\mu_{\mathrm{rel}}}, \theta_{\mathrm{E}}=\frac{r_{\mathrm{E}}}{D_{\mathrm{d}}}, \mu_{\mathrm{rel}} \equiv \frac{v_{\mathrm{t}}}{D_{\mathrm{d}}}
$$

where $v_{\mathrm{t}}$ is the transverse velocity and $\mu_{\text {rel }}$ is the relative lens-source proper motion. Substituting the expression for the Einstein radius into eq. (3.11), we find that

$$
t_{\mathrm{E}} \approx 19 \text { day } \sqrt{4 \times \frac{D_{\mathrm{d}}}{D_{\mathrm{s}}}\left(1-\frac{D_{\mathrm{d}}}{D_{\mathrm{s}}}\right)}\left(\frac{D_{\mathrm{s}}}{8 \mathrm{kpc}}\right)^{1 / 2}\left(\frac{M}{0.3 M_{\odot}}\right)^{1 / 2}\left(\frac{v_{\mathrm{t}}}{200 \mathrm{~km} \mathrm{~s}^{-1}}\right)^{-1 / 2} .
$$

If the closest approach is achieved a time $t=t_{0}$, then the dimensionless coordinates are $x_{\mathrm{S}}=(t-$ $\left.t_{0}\right) / t_{\mathrm{E}}$ and $y_{\mathrm{s}}=u_{0}$, and the magnification as a function of time is given by

$$
\mu(t)=\frac{r_{\mathrm{s}}^{2}(t)+2}{r_{\mathrm{s}}(t) \sqrt{r_{\mathrm{s}}^{2}(t)+4}}, \quad r_{\mathrm{s}}(t)=\sqrt{u_{0}^{2}+\left(\frac{t-t_{0}}{t_{\mathrm{E}}}\right)^{2}} .
$$

Two light curve examples are shown in the right panel of Fig. 1 for $u_{0}=0.1$ and 0.3 .

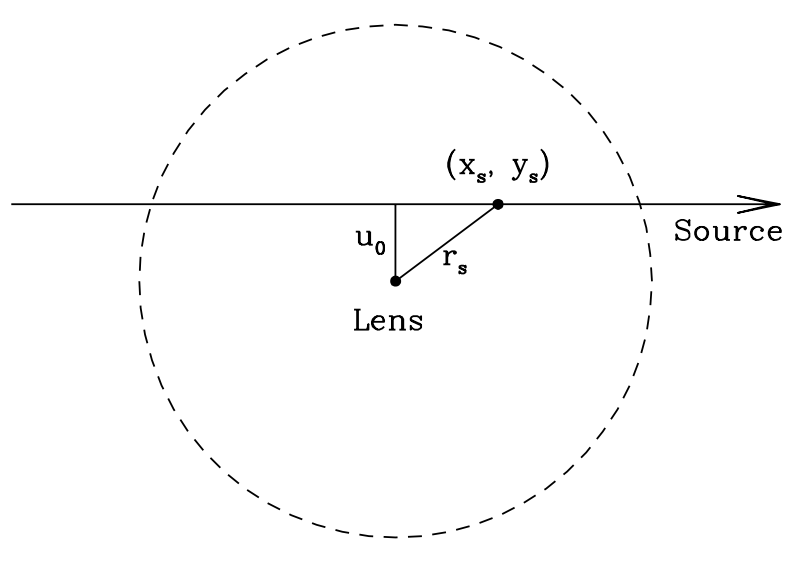

Figure 4: Illustration for the lens position and source trajectory. The dimensionless impact parameter is $u_{0}$, $\left(x_{\mathrm{s}}, y_{\mathrm{s}}\right)$ are the dimensionless source position along the trajectory, and $r_{\mathrm{s}}$ is the distance between the lens and source.

To model an observed light curve, three parameters are present in eq. (4.3): $t_{0}, t_{\mathrm{E}}, u_{0}$. In practise, we need two additional parameters, $m_{0}$, the baseline magnitude, and $f_{\mathrm{s}}$, a blending parameter. $f_{\mathrm{s}}$ characterises the fraction of light contributed by the lensed source; in crowded stellar fields, each observed 'star' may be a composite of the lensed star, other unrelated stars within the seeing disk and the lens if it is luminous $([2,31])$. Blending will lower the observed magnification and in general $f_{\mathrm{s}}$ depends on the wavelength, and so each filter requires a separate $f_{\mathrm{s}}$ parameter. Unfortunately, we can see from eq. (4.3) that there is only one physical parameter $\left(t_{\mathrm{E}}\right)$ in the model that relates to the lens properties. $t_{\mathrm{E}}$ depends on the lens mass, distances to the lens and source, 
and the transverse velocity $\mu_{\text {rel }}$. Therefore from an observed light curve well fitted by the standard model, one cannot infer the lens distance and mass uniquely; this is the so-called microlensing degeneracy. However, given a lens mass function and some kinematic model of the Milky Way, we can infer the lens mass statistically.

\subsection{Non-standard light curves}

The standard model assumes the lensed source is point-like, both the lens and source are single and all the motions are linear. The majority $(\sim 90 \%)$ of microlensing events are well described by this simple model. However, about $10 \%$ of the light curves are non-standard (exotic), due to the breakdown of one (or more) of the assumptions. We briefly list these possibilities below (see the talk by Dominik for more details.) These non-standard microlensing events allow us to derive extra constraints, and partially lift the microlensing degeneracy. Because of this, they play a role far greater than their numbers suggest.

(1) The lens may be in a binary or even a multiple system ([34]). The light curves for a binary or multiple lensing system can be much more diverse (see 4.3). They offer an exciting way to discover extrasolar planets $([34,26,8,29,41])$.

(2) The source is in a binary. In this case, the light curve will be a simple, linear superposition of the two sources (when the rotation can be neglected, see [28]).

(3) The finite size of the lensed star cannot be neglected. This occurs when the impact parameter $u_{0}$ is comparable to the stellar radius normalised to the Einstein radius, $u_{0} \sim r_{\star} / r_{\mathrm{E}}$. In this case, the light curve is significantly modified by the finite source size effect $([53,22])$. The finite source size effect is most important for high magnification events.

(4) The standard light curve assumes all the motions are linear. However, the source and/or the lens may be in a binary, furthermore, the Earth rotates around the Sun. All these motions induce accelerations. The effect due to the Earth motion around the Sun is usually called "parallax" (e.g. [21, 48, 39]), while that due to binary motion in the source plane is called "xallarap" ("parallax" spelt backwards, $[7,1])$. Parallax or "xallarap" events usually have long timescales. For a typical microlensing event with timescale $t_{\mathrm{E}} \sim 20$ day, the parallax effect due to the Earth rotation around the Sun is often undetectable (unless the photometric accuracy of the light curve is very high).

(5) Microlensing can "repeat", in particular if the lens is a wide binary ([14]) or the source is a wide binary. In such cases, microlensing may manifest as two well-separated peaks, i.e., as a "repeating" event. A few percent of microlensing events are predicted to repeat, consistent with the observations ([47]).

Notice that several violations may occur at the same time, which in some cases allow the microlensing degeneracy to be completely removed (e.g. $[4,17,20])$. 


\section{3 $N$-point lens gravitational microlensing}

It is straightforward to derive the (dimensionless) lens equation for $N$-point lenses. We can first cast eq. (3.8) in vector form and then rearrange

$$
\overrightarrow{r_{\mathrm{S}}}=\vec{r}-\frac{1}{|\vec{r}|^{2}} \vec{r}
$$

The above expression implicitly assumes that the lens is at the origin, and all the lengths have been normalised to the Einstein radius corresponding to its mass (or equivalently, the lens mass has been assumed to be unity).

Let us consider the general case where we have $N$-point lenses, at $\vec{r}_{k}=\left(x_{k}, y_{k}\right)$ with mass $M_{k}$, $k=1, \cdots, N$. We normalise all the lengths with the Einstein radius corresponding to the total mass, $M=\sum_{k=1}^{N} M_{k}$. The generalised lens equation then reads

$$
\vec{r}_{\mathrm{s}}=\vec{r}-\sum_{k=1}^{N} m_{k} \frac{\vec{r}-\vec{r}_{k}}{\left|\left(\vec{r}-\vec{r}_{k}\right)\right|^{2}}, \quad m_{k}=\frac{M_{k}}{M}
$$

where $\sum_{k=1}^{N} m_{k}=1$. If there is only one lens $\left(m_{1}=1\right)$ and the lens is at the origin, then eq. (4.5) reverts to the single lens equation (4.4).

Two-dimensional vectors and complex numbers are closely related, Witt (1990) first demonstrated that the above equation can be cast into a complex form by direct substitutions of the vectors by complex numbers:

$$
z_{\mathrm{s}}=z-\sum_{k=1}^{N} m_{k} \frac{z-z_{k}}{\left(z-z_{k}\right)\left(\bar{z}-\bar{z}_{k}\right)}=z-\sum_{k=1}^{N} \frac{m_{k}}{\bar{z}-\bar{z}_{k}}
$$

where $z=x+y i, z_{k}=x_{k}+y_{k} i$, and $z_{\mathrm{s}}=x_{\mathrm{s}}+y_{\mathrm{s}} i$ (where $i$ is the imaginary unit).

We can take the conjugate of eq. (4.6) and obtain an expression for $\bar{z}$. Substituting this back into eq. (4.6), we obtain a complex polynomial of degree $n^{2}+1$. This immediately shows that even a binary lens equation cannot be solved analytically since it is a fifth-order polynomial ${ }^{7}$.

The magnification is related to the determinant of the Jacobian of the mapping from the source plane to the lens plane: $\left(x_{\mathrm{s}}, y_{\mathrm{s}}\right) \rightarrow(x, y)$. In the complex form, this is ([52]):

$$
\mu=J^{-1}, \quad J=\frac{\partial\left(x_{\mathrm{s}}, y_{\mathrm{s}}\right)}{\partial(x, y)}=1-\frac{\partial z_{\mathrm{s}}}{\partial \bar{z}} \frac{\overline{\partial z_{\mathrm{s}}}}{\partial \bar{z}} .
$$

Notice that the Jacobian can be equal to zero implying a (point) source will be infinitely magnified. The image positions satisfying $J=0$ form continuous critical curves, which are mapped into caustics in the source plane. Of course, stars are not point-like, they have finite sizes. The finite source size of a star smoothes out the singularity. As a result, the magnification remains finite.

For $N$-point lenses, from the complex lens equation (4.6), we have

$$
\frac{\partial z_{\mathrm{s}}}{\partial \bar{z}}=\sum_{k=1}^{N} \frac{m_{k}}{\left(\bar{z}-\bar{z}_{k}\right)^{2}}, \quad J=1-\left|\sum_{k=1}^{N} \frac{m_{k}}{\left(\bar{z}-\bar{z}_{k}\right)^{2}}\right|^{2} .
$$

\footnotetext{
${ }^{7}$ In classical mechanics, the two-body problem can be solved analytically, but not the three-body problem.
} 

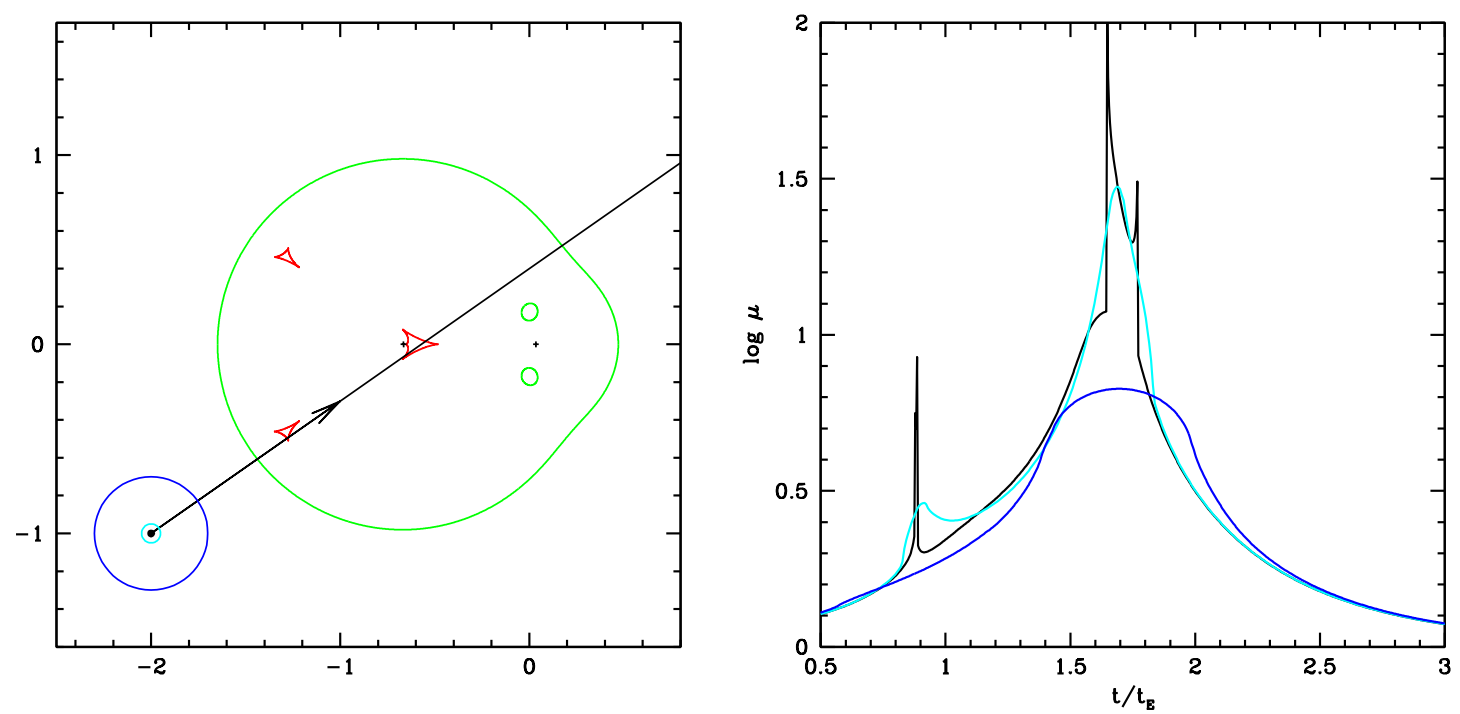

Figure 5: Left panel: Caustics (red curves) and critical curves (green curves) for a binary lens. The lenses (indicated by two ' + ' signs) are located at $z_{1}=(0.665,0)$ and $z_{2}=(0.035,0)$ with mass $m_{1}=0.95$ and and $m_{2}=1-m_{1}=0.05$ respectively. The green line shows the trajectory for three source sizes, $r_{\mathrm{s}} / r_{\mathrm{E}}=$ $0,0.05,0.3$, indicated by the cyan and blue circles and a dot (for a point source). The trajectory starts at $(-2,-1)$ with a slope of 0.7. Right panel: Corresponding light curves for the three source sizes along the trajectory in the left panel. The time is normalised to the Einstein radius crossing time, $t_{\mathrm{E}}$, and $t=0$ corresponds to the starting position. Notice that as the source size increases, the lensing magnification amplitude decreases.

It follows that the critical curves are given by

$$
\left|\sum_{k=1}^{N} \frac{m_{k}}{\left(\bar{z}-\bar{z}_{k}\right)^{2}}\right|^{2}=1 .
$$

The sum in the above equation must be on a unit circle, and the solution can be cast in a parametric form

$$
\sum_{k=1}^{N} \frac{m_{k}}{\left(z-z_{k}\right)^{2}}=\mathrm{e}^{i \Phi}
$$

where $0 \leq \Phi<2 \pi$ is a parameter. The above equation is a complex polynomial of degree of $2 N$ with respect to $z$. For each $\Phi$, there are at most $2 N$ distinct solutions. As we vary $\Phi$ continuously, the solutions trace out at most $2 N$ continuous critical curves (critical curves of different solutions may join with each other smoothly). In practise, we can solve the equation for one $\Phi$ value, and then use the Newton-Raphson method to find the solutions for other values of $\Phi$.

For a single point lens, if we take $z_{1}=0$, and $m_{1}=1$, we find that the critical curve is the Einstein ring $(|z|=1)$, which is mapped into a degenerate caustic point at the origin $\left(z_{\mathrm{s}}=0\right)$. However, for binary or multiple lenses, the critical curves and caustics are much more complex. The left panel in Fig. 5 illustrates the critical curves and caustics for a binary lens with $m_{1}=0.95$ and $m_{2}=0.05$ and separation of 0.7 (in units of the Einstein radius for the total mass). In this case, there are three separate, continuous critical curves which are mapped into three caustics.

For a point source, the complex polynomial can be easily solved numerically (e.g. using the zroots routine in [40]). However, for a source with finite size, the existence of singularities 
makes the integration time-consuming (see section 6). The right panel in Fig. 5 shows the light curves for three source sizes along the trajectory indicated by the green straight line. As the source size increases, the lensing magnification amplitude decreases, and the number of peaks differ for different source sizes.

\section{Optical depth and event rates}

So far we have derived the lens equation and light curve for microlensing by a single star. In reality, hundreds of millions of stars are monitored, and $\approx 800$ unique microlensing events are discovered each year. Clearly we need some statistical quantities to describe microlensing experiments. For this, we need two key concepts: optical depth and event rate.

\subsection{Optical depth}

The optical depth (lensing probability) is the probability that a given source falls into the Einstein radius of any lensing star along the line of sight. Thus the optical depth can be expressed as

$$
\tau=\int_{0}^{D_{\mathrm{s}}} n\left(D_{\mathrm{d}}\right)\left(\pi r_{\mathrm{E}}^{2}\right) d D_{\mathrm{d}}
$$

which is an integral of the product of the number density of lenses, the lensing cross-section (= $\left.\pi r_{\mathrm{E}}^{2}\right)$ and the differential path $\left(d D_{\mathrm{d}}\right)$.

Alternatively, the optical depth can be viewed as the fraction of sky covered by the angular areas of all the lenses, which yields another expression

$$
\tau=\frac{1}{4 \pi} \int_{0}^{D_{\mathrm{s}}}\left[n\left(D_{\mathrm{d}}\right) 4 \pi D_{\mathrm{d}}^{2} d D_{\mathrm{d}}\right]\left(\pi \theta_{\mathrm{E}}^{2}\right)
$$

where the term in the [ ] gives the numbers of lenses in a spherical shell with radius $D_{\mathrm{d}}$ to $D_{\mathrm{d}}+d D_{\mathrm{d}}$, $\pi \theta_{\mathrm{E}}{ }^{2}$ is the angular area covered by a single lens, and the term in the denominator is the total solid angle over all the sky $(4 \pi)$.

If all the lenses have the same mass $M$, then $n\left(D_{\mathrm{d}}\right)=\rho\left(D_{\mathrm{d}}\right) / M, \pi r_{\mathrm{E}}^{2} \propto M$, and the lens mass drops out in $n\left(D_{\mathrm{d}}\right) \pi r_{\mathrm{E}}{ }^{2}$. Therefore the optical depth depends on the total mass density along the line of sight, but not on the mass function.

Let us consider a simple model where the density is constant along the line of sight, $\rho\left(D_{\mathrm{d}}\right)=$ $\rho_{0}$. Integrating along the line of sight one finds

$$
\tau=\frac{2 \pi G}{3 c^{2}} D_{\mathrm{s}}^{2}=\frac{1}{2 c^{2}} \frac{G \rho_{0} 4 \pi D_{\mathrm{s}}{ }^{3} / 3}{D_{\mathrm{s}}}=\frac{1}{2 c^{2}} \frac{G M\left(<D_{\mathrm{s}}\right)}{D_{\mathrm{s}}}=\frac{V^{2}}{2 c^{2}},
$$

where $M\left(<D_{\mathrm{s}}\right)$ is the total mass enclosed within the sphere of radius $D_{\mathrm{s}}$ and the circular velocity is given by $V^{2}=G M\left(<D_{\mathrm{s}}\right) / D_{\mathrm{s}}$.

For the Milky Way, $V \approx 200 \mathrm{~km} \mathrm{~s}^{-1}, \tau \approx 5 \times 10^{-7}$. The low optical depth means millions of stars have to be monitored to have a realistic yield of microlensing events, and thus one needs to observe dense stellar fields, which in turn means accurate crowded field photometry is essential (see the talk by P. Wozniak on difference image analysis). 


\subsection{Event rate}

The optical depth indicates the probability of a given star that is within the Einstein radii of the lenses at any given instant. As such, the optical depth is a static concept. We are obviously interested in knowing the event rate (a dynamic concept), i.e., the number of (new) microlensing events per unit time for a given number of monitored stars, $N_{\star}$.

To calculate the event rate, it is easier to imagine the lenses are moving in a static stellar source background. For simplicity, let us assume all the lens move with the same velocity of $v_{\mathrm{t}}$. The new area swept out by each lens in the time interval $d t$ is equal to the product of the diameter of the Einstein ring and the distance travelled $v_{\mathrm{t}} d t, d A=2 r_{\mathrm{E}} \times v_{\mathrm{t}} d t=2 r_{\mathrm{E}}^{2} d t / t_{\mathrm{E}}$. The probability of a source becoming a new microlensing event is given by

$$
d \tau=\int_{0}^{D_{\mathrm{s}}} n\left(D_{\mathrm{d}}\right) d A d D_{\mathrm{d}}=\int_{0}^{D_{\mathrm{s}}} n\left(D_{\mathrm{d}}\right)\left(\frac{2 r_{\mathrm{E}}^{2}}{t_{\mathrm{E}}}\right) d t d D_{\mathrm{d}}
$$

The total number of new events is $N_{\star} d \tau$, and thus the event rate is given by

$$
\Gamma=\frac{d\left(N_{\star} \tau\right)}{d t}=N_{\star} \int_{0}^{D_{\mathrm{s}}} n\left(D_{\mathrm{d}}\right)\left(\frac{2}{\pi t_{\mathrm{E}}} \cdot \pi r_{\mathrm{E}}^{2}\right) d D_{\mathrm{d}}=\frac{2 N_{\star}}{\pi} \int_{0}^{D_{\mathrm{s}}} \frac{d \tau}{t_{\mathrm{E}}} .
$$

If, for simplicity, we assume all the Einstein radius crossing times are identical, then we have

$$
\Gamma \approx \frac{2 N_{\star}}{\pi} \frac{\tau}{t_{\mathrm{E}}} .
$$

We make several remarks about the event rate:

(1) If we take $t_{\mathrm{E}}=19$ day (roughly equal to the median of the observed timescales), then we have

$$
\Gamma \approx \frac{2 N_{\star}}{\pi} \frac{\tau}{t_{\mathrm{E}}}=1200 \mathrm{yr}^{-1} \frac{N_{\star}}{10^{8}} \frac{\tau}{10^{-6}}\left(\frac{t_{\mathrm{E}}}{19 \text { day }}\right)^{-1}
$$

For OGLE-III, about $2 \times 10^{8}$ stars are monitored (see Udalski's contribution), so the total number of events lenses we expect per year is $\Gamma \sim 2400$ if $\tau \sim 10^{-6}$, which is a factor of four of the observed rate (indicating the detection efficiency may be of the order of $30 \%$ ).

(2) While the optical depth does not depend on the mass function, the event rate does because of $t_{\mathrm{E}}\left(\propto M^{1 / 2}\right)$ in the denominator of eq. (5.6). The timescale distribution can be used to probe the kinematics and mass function of lenses in the Milky Way.

(3) The lenses and sources have velocity distributions, one must account for them when realistic event rates are needed. Furthermore, the source distance is unknown, and so in general we need to average over the source distance (for example calculations, see [27, 30]).

\section{Summary}

In this introduction, we derived the lens equation, and obtained the image positions and magnifications for a point lens. We also discussed the statistical measures for microlensing experiments, and estimated the order of magnitudes for various quantities. An interested reader should now be 
armed with a basic knowledge of microlensing and be prepared to read the review articles and start to do research on gravitational microlensing (or even try to solve the problem set below).

Since the discovery of first microlensing events in the early 1990's, enormous achievements have been made in the field. However, challenges and opportunities remain.

(1) Undoubtedly the highlight of gravitational microlensing in the last few years has been the discovery of extrasolar planets $([11,50,5,23,10])$. Microlensing has much to offer in this area since it probes a different part of the parameter space, and provides an important test of the core accretion theory of planet formation. Several White Papers ([24, 9, 16, 6]) set out strategies with ambitious milestones in the next fifteen years, from improvement of the current survey plus followup mode of discovery (with an automated algorithm to identify the "anomalies" in real-time) in the near term, to a wide-field network from the ground in the next 5-10 years, and eventually a telescope in space in the next 10-15 years. Combined with the stellar transit mission Kepler (to be launched in 2009), microlensing will be able to provide the complete census of Earth-mass (and lower) planets at virtually all the separations.

Technically, it is still challenging to calculate the light curves for sources with finite size since we need to integrate over the singularities of caustics. This is particularly important for the discovery of extrasolar planets when a source transits the small caustics induced by the planet(s). The problem becomes even worse with the discovery of multiple planets ([20]) due to the higher complexity of the lens equation and the increased number of parameters: how do we search the high dimensional parameter space efficiently?

Are there hidden multiple planetary light curves in the database that are not yet identified due to their complex shapes?

(2) Microlensing surveys over the last fifteen years have accumulated tens of TB of data. This tremendous database has not been exploited to its fullest potential.

For example, the surveys yielded many high-quality colour-magnitude diagrams of stellar popular populations, proper motions of millions of stars, and in the future the optical depth maps. All these can be used to provide important and independent probes of the structure of the Milky Way.

Despite promising earlier attempts (e.g. [36]; [43, 44]; [30, 55]), microlensing has underdelivered in this area. For example, while we have discovered several thousands of microlensing events over the last 15 years, only a small fraction has been used for statistical analyses of optical depths. We need to remedy the situation urgently.

(3) High-magnification events are great targets-of-opportunity for high signal-to-noise ratio spectroscopic observations to study stellar atmospheres for bulge stars. Attempts so far already yielded interesting results (e.g. [32, 49, 12]). We need to explore this more systematically.

(4) For mathematically-gifted students (or mathematicians), gravitational microlensing provides an interesting problem. While the binary lens equation is no longer analytical, there is, nevertheless, an analytical relation on the minimum magnification for five-image configurations $([54,46])$. There is also a degeneracy found by Dominik (1999) between close and wide 
separation binaries, which was later explored in much greater detail by An (2005). Are there any other symmetries, perhaps even for multiple lens systems?

The number of critical curves for $N$-point lenses cannot exceed $2 N$ (see section 4.3). The upper bound of the number of images for $N$-point lenses also has linear dependence on $N$ (see Problem 1). Are these two related in some geometric way?

\section{References}

[1] Alcock C. et al. 2001, ApJ, 552, 259

[2] Alcock C. et al. 2001, Nature, 414, 617

[3] An J. H. 2005, MNRAS, 356, 1409

[4] An J. H. et al. 2002, ApJ, 572, 521

[5] Beaulieu J. P. et al. 2006, Nature, 439, 437

[6] Beaulieu J. P., Kerins E., Mao, S. et al. 2008, arXiv:0808.0005 (White Paper Submitted ESA's Exo-Planet Roadmap Advisory Team)

[7] Bennett D. P. 1998, Physics Reports, 307, 97

[8] Bennett D. P., Rhie, S. H. 1996, ApJ, 472, 660

[9] Bennett D. P., Anderson J. Beaulieu J. P. et al. 2007, arXiv:0704.0454 (White Paper Submitted to the NASA/NSF Exoplanet Task Force)

[10] Bennett D. P. et al. 2008, arXiv0806.0025

[11] Bond I. A. et al. 2004, ApJ, 606, L155

[12] Cohen J. G., Huang W. J., Udalski A., Gould A. Johnson, J. A. 2008, ApJ, 682, 1029

[13] Delplancke F., Górski K. M., Richichi A. 2001, A\&A, 375, 701

[14] di Stefano R., Mao S. 1996, ApJ, 457, 93

[15] Dominik M. 1999, A\&A, 349, 108

[16] Dominik M. et al. 2008, arXiv:0808.0004 (White Paper Submitted ESA's Exo-Planet Roadmap Advisory Team)

[17] Dong S. B. et al. 2008, 2008arXiv0804.1354

[18] Einstein A. 1936, Science, 84, 506

[19] Evans N. W. 2003, in "Gravitational Lensing: A Unique Tool For Cosmology", eds D. Valls-Gabaud, J.-P. Kneib (arXiv:astro-ph/0304252v2)

[20] Gaudi S. et al. 2008, Science, 319, 927

[21] Gould A. 1992, ApJ, 392, 442

[22] Gould A. 1994, ApJ, 421, L71

[23] Gould A. et al. 2006, ApJ, 644, L37

[24] Gould A., Gaudi B. S., Bennett D. P., et al. 2007, arXiv:0704.0767 (White Paper Submitted to the NASA/NSF Exoplanet Task Force) 
[25] Gould A. 2008a, in the "The Variable Universe: A Celebration of Bohdan Paczynski", arXiv:0803.4324

[26] Gould A., Loeb A. 1992, ApJ, 396, 104

[27] Griest K. 1991, ApJ, 366, 412

[28] Griest K., Hu W. 1992, ApJ, 397, 362

[29] Griest K., Safizadeh N. 1998, ApJ, 500, 37

[30] Kiraga M., Paczyński 1994, ApJ, 430, L101

[31] Kozłowski S., Woźniak P. R., Mao S., Wood A. 2007, 671, 420

[32] Lennon D. J., Mao S., Fuhrmann K., Gehren T. 1996, ApJ, 471, L23

[33] Liebes S. 1964, Physical Review, 133, 835

[34] Mao S., Paczyński, B. 1991, ApJ, 374, L37

[35] Mao S. 2001 in "Gravitational Lensing: Recent Progress and Future Goals”, ASP Conference Proceedings, 237, eds. T. G. Brainerd and C. S. Kochanek, p. 237

[36] Ng Y. K., Bertelli G., Chiosi C., Bressan A. 1996, A\&A, 310, 771

[37] Paczyński B. 1986, ApJ, 304, 1

[38] Paczyński B. 1996, ARA\&A, 34, 419

[39] Poindexter S., Afonso, C. Bennett D. P., Glicenstein J.-F., Gould A., Szymański M. K., Udalski A. 2005, ApJ, 633, 914

[40] Press W. H., Teukolsky S. A., Vetterling W. T., Flannerty B.P. 1992, Numerical Recipes in C: The Art of Scientific Computing (2nd Edition) (Cambridge University Press: New York)

[41] Rattenbury N. J., Bond I. A., Skuljan J., Yock P. C. M. 2002, MNRAS, 335, 159

[42] Rattenbury N. J., Mao S. 2006, MNRAS, 365, 792

[43] Rattenbury N. J., Mao S., Debattista V. P., Sumi T., Gerhard O., de Lorenzi F. 2007, MNRAS, 378, 1165

[44] Rattenbury N. J., Mao S., Sumi T., Smith M. C. 2007, MNRAS, 378, 1064

[45] Refsdal, S. 1964, MNRAS, 128, 295

[46] Rhie S. H. 1997, ApJ, 484, 63

[47] Skowron J., Wyrzykowski Ł, Mao S., Jaroszyński M. 2008, in preparation

[48] Smith M. C., Mao S. Woźniak P. 2002, MNRAS, 332, 962

[49] Thurl C., Sackett P. D., Hauschildt P. H. 2006, A\&A, 455, 315

[50] Udalski, A. et al. 2005, ApJ, 628, L109

[51] Wambganss J. 2006, in “Gravitational lensing: strong, weak and micro”, eds. G. Meylan, P. Jetzer and P. North, p. 453

[52] Witt H. J. 1990, A\&A, 236, 311

[53] Witt H. J., Mao S. 1994, ApJ, 430, 505

[54] Witt H. J., Mao S. 1995, ApJ, 447, L105

[55] Zhao H. S., Rich R. M., Spergel D. N. 1996, MNRAS, 282, 175 


\section{Problems}

1. a) There are $N$-point lenses in a lens plane. For a source very far away from all the lenses, how many images are there? What are their parities?

b) What is the achievable maximum number of images for $N$-point lenses and an arbitrary source position?

2. A uniform source star is perfectly aligned with an observer and a point lens. Its physical radius normalised to the Einstein radius is $\rho_{\star}$.

a) What is the resulting image configuration?

b) What is the magnification for the finite source?

c) Estimate the maximum magnification that can be achieved for a source star in the Galactic bulge.

d) Derive the expression for magnification when the source is not perfectly aligned with the lens.

3. Show that the total magnification for a point lens is always larger than one. How can this be reconciled with energy conservation? (see Jaroszyński \& Paczynski 1996, AcA, 46, 361).

4. The density distribution in the plane of the Galactic disk can be modelled as an exponential

$$
\rho(R)=\rho_{0} \exp \left(-\left(R-R_{0}\right) / R_{d}\right)
$$

where $\rho_{0}$ is the density in the solar neighbourhood, $R_{0}$ is the distance to the Galactic centre, $R_{d}$ is the disk scale length, and $R$ is the distance from the Galactic centre.

a) Find the optical depth $\tau$ for a source at the Galactic centre $(R=0)$.

b) If $\rho_{0}=0.1 M_{\odot} \mathrm{pc}^{-3}, R_{0}=8 \mathrm{kpc}, R_{d}=3 \mathrm{kpc}$, what is the value of $\tau$ ?

5. Consider a simple model: all lensing objects have the same mass $M$, the same three-dimensional velocity $V$, and their velocity vector directions have an isotropic distribution. The source located at the distance is stationary, and the number density of lensing objects is uniform between the observer and the source. Derive the timescale probability distribution.

Now assume the lenses follow a Maxwellian distribution with a one-dimensional velocity dispersion $\sigma$. Derive the timescale probability distribution. Show that it follows a power-law behaviour for both very short and very long timescales.

6. A distance source is lensed by a point deflector with mass $M$. The light signals emitted by the source will be received by an observer at different times for the two images due to the difference in the trajectory and gravitational potential experienced. The time delay is of the order of $r_{\mathrm{sch}} / c$, where $r_{\mathrm{sch}}$ is the Schwarzschild radius. Is this observable for a $M=1 M_{\odot}$ lens?

7. A background star stationary at the origin is microlensed by a lens moving from $-\infty$ to $\infty$. Show that the centre of light of the two images traces out an ellipse. 
8. Show that

a) In the complex notation, the Jacobian is given by eq. (4.8).

b) For any positive-parity image produced by $N$-point lenses, the magnification is always larger than or equal to one.

c) Find the number and positions of images where their magnifications are identical to unity for a binary lens.

d) What is the maximum number images with unity magnification for $N$-point lenses? 


\section{Solutions}

1. a) There are $N+1$ images, one bright image close to the source, and $N$ fainter images with one image close to each lens. The image close to the source has positive parity, all the others have negative parities.

b) $5(N-1)$ for $N \geq 2$. This is a difficult problem. See Rhie S. H. 2003, arXiv:astroph/0305166; Khavinson, D. and Neumann G. 2006, Proceedings Of the American Mathematical Society, 134, 1077 (arXiv:math/04011088).

2. a) The image configuration is an annulus. The inner radius is given by the $r_{2}=\left(\sqrt{\rho_{\star}^{2}+4}+\right.$ $\left.\rho_{\star}\right) / 2$ while the outer radius is given by $r_{1}=\left(\sqrt{\rho_{\star}^{2}+4}-\rho_{\star}\right) / 2$.

b) The area covered by the image is $A=\pi\left(r_{2}^{2}-r_{1}^{2}\right)=\pi \rho_{\star} \sqrt{\rho_{\star}^{2}+4}$. Thus the magnification is $A /\left(\pi \rho_{\star}^{2}\right)=\sqrt{\rho_{\star}^{2}+4} / \rho_{\star}$. In particular, when $\rho_{\star} \rightarrow 0, A \rightarrow 2 / \rho_{\star}$.

c) For a solar mass lens in the Galactic centre $\left(D_{\mathrm{s}}=8 \mathrm{AU}\right)$, the maximum Einstein radius is achieved when $D_{\mathrm{d}}=D_{\mathrm{s}} / 2, r_{\mathrm{E}}=6.0 \times 10^{13} \mathrm{~cm}$. The faintest and smallest stars we can see in the bulge have radii similar to a solar-type star, $r_{\star} \approx R_{\odot}=7 \times 10^{10} \mathrm{~cm}, \rho_{\star}=$ $r_{\star} / r_{E}=1.2 \times 10^{-3}$. The maximum magnification is of the order of $\approx 2 / \rho_{\star} \approx 1700$.

d) See Witt \& Mao 1994, ApJ, 430, 505

3. Clearly $\mu>1$. See Jaroszyński \& Paczynski 1996, AcA, 46, 361 for discussions about the energy conservation.

4. The optical depth is given by

$$
\tau=\int_{0}^{D_{\mathrm{s}}} n\left(D_{\mathrm{d}}\right)\left(\pi r_{\mathrm{E}}^{2}\right) d D_{\mathrm{d}}=\int_{0}^{D_{\mathrm{s}}} \frac{\rho\left(D_{\mathrm{d}}\right)}{M} \frac{4 \pi G M}{c^{2}} \frac{D_{\mathrm{d}} D_{\mathrm{ds}}}{D_{\mathrm{s}}} d D_{\mathrm{d}} .
$$

For microlensing in the Galactic plane, $D_{\mathrm{ds}}=R, D_{\mathrm{s}}=R_{0}, D_{\mathrm{d}}=R_{0}-R$, and thus we have

$$
\tau=\int_{0}^{R_{0}} \rho_{0} \mathrm{e}^{D_{\mathrm{d}} / R_{d}} \frac{4 \pi G}{c^{2}} \frac{D_{\mathrm{d}}\left(R_{0}-D_{\mathrm{d}}\right)}{R_{0}} d D_{\mathrm{d}}
$$

Performing the above integral, we find that

$$
\tau=\frac{4 G \pi \rho_{0} R_{0}^{2}}{c^{2}} y^{-3}\left[2+y+\mathrm{e}^{y}(-2+y)\right], \quad y=R_{0} / R_{d}
$$

For the given numbers, we find $4 \pi G \rho_{0} R_{0}^{2} / c^{2}=3.86 \times 10^{-6}$, and $y=R_{0} / R_{d}=2.67$, we have

$$
\tau \approx 2.9 \times 10^{-6}
$$

5. For step by step derivations, see Ma \& Paczyński, 1996, ApJ, 473, 57. Notice that the observed event timescale distribution does not follow the power-laws due to detection efficiency.

6. The time delay is of the order of tens of micro-seconds for a solar mass lens, and is very difficult to observe. 
7. See Hog et al. (1995, A\&A, 294, 287), Miyamoto \& Yoshi (1995, AJ, 110, 1427), Walker (1995, ApJ, 453, 37)

8. a) Using complex notation, we have $x=\frac{1}{2}(z+\bar{z}), y=\frac{1}{2 i}(z-\bar{z})$. Thus

$$
\frac{\partial z_{\mathrm{s}}}{\partial z}=\frac{\partial z_{\mathrm{s}}}{\partial x} \frac{\partial x}{\partial z}+\frac{\partial z_{\mathrm{s}}}{\partial y} \frac{\partial y}{\partial z}=\frac{1}{2}\left(\frac{\partial z_{\mathrm{s}}}{\partial x}-i \frac{\partial z_{\mathrm{s}}}{\partial y}\right)=\frac{1}{2}\left(\frac{\partial x_{\mathrm{s}}}{\partial x}+i \frac{\partial y_{\mathrm{s}}}{\partial x}-i \frac{\partial x_{\mathrm{s}}}{\partial y}+\frac{\partial y_{\mathrm{s}}}{\partial y}\right) .
$$

Similarly

$$
\frac{\partial z_{\mathrm{s}}}{\partial \bar{z}}=\frac{\partial z_{\mathrm{s}}}{\partial x} \frac{\partial x}{\partial \bar{z}}+\frac{\partial z_{\mathrm{s}}}{\partial y} \frac{\partial y}{\partial \bar{z}}=\frac{1}{2}\left(\frac{\partial z_{\mathrm{s}}}{\partial x}+i \frac{\partial z_{\mathrm{s}}}{\partial y}\right)=\frac{1}{2}\left(\frac{\partial x_{\mathrm{s}}}{\partial x}+i \frac{\partial y_{\mathrm{s}}}{\partial x}+i \frac{\partial x_{\mathrm{s}}}{\partial y}-\frac{\partial y_{\mathrm{s}}}{\partial y}\right)
$$

However, from the lens equation (4.6), $\partial z_{\mathrm{s}} / \partial z=1$, and thus comparing the real and imaginary parts in eq. (6.5), we have

$$
\frac{\partial x_{\mathrm{s}}}{\partial x}+\frac{\partial y_{\mathrm{s}}}{\partial y}=1, \quad \frac{\partial x_{\mathrm{s}}}{\partial y}=\frac{\partial y_{\mathrm{s}}}{\partial x}
$$

Substituting the second expression into eq. (6.6), we find

$$
\frac{\partial z_{\mathrm{s}}}{\partial \bar{z}}=\frac{1}{2}\left(\frac{\partial x_{\mathrm{s}}}{\partial x}-\frac{\partial y_{\mathrm{s}}}{\partial y}\right)+i \frac{\partial y_{\mathrm{s}}}{\partial x}
$$

Combined with

$$
\frac{\partial z_{\mathrm{s}}}{\partial z}=\frac{1}{2}\left(\frac{\partial x_{\mathrm{s}}}{\partial x}+\frac{\partial y_{\mathrm{s}}}{\partial y}\right)=1
$$

we find

$$
\frac{\partial x_{\mathrm{s}}}{\partial x}=1+\frac{1}{2}\left(\frac{\partial z_{\mathrm{s}}}{\partial \bar{z}}+\frac{\overline{\partial z_{\mathrm{s}}}}{\partial \bar{z}}\right), \frac{\partial y_{\mathrm{s}}}{\partial y}=1-\frac{1}{2}\left(\frac{\partial z_{\mathrm{s}}}{\partial \bar{z}}+\frac{\overline{\partial z_{\mathrm{s}}}}{\partial \bar{z}}\right), \frac{\partial y_{\mathrm{s}}}{\partial x}=\frac{1}{2 i}\left(\frac{\partial z_{\mathrm{s}}}{\partial \bar{z}}-\overline{\frac{\partial z_{\mathrm{s}}}{\partial \bar{z}}}\right)
$$

Substituting the above equations into the Jacobian

$$
J=\frac{\partial x_{\mathrm{s}}}{\partial x} \frac{\partial y_{\mathrm{s}}}{\partial y}-\frac{\partial y_{\mathrm{s}}}{\partial x} \frac{\partial x_{\mathrm{s}}}{\partial y}
$$

we recover the required expression.

b) Since

$$
J=1-\left|\sum_{k=1}^{N} \frac{m_{k}}{\left(\bar{z}-\bar{z}_{k}\right)^{2}}\right|^{2} .
$$

For a positive parity image, we must have $1 \geq J>0$, it follows that $\mu=J^{-1} \geq 1$. The magnification is unity when the sum is equal to zero.

c) Without losing generality, we put the lenses on the $x$-axis, at $\left(z_{0}, 0\right)$ and $\left(-z_{0}, 0\right)$, with masses $m_{1}$ and $m_{2}\left(m_{1} \geq m_{2}\right)$, and we have $m_{1}+m_{2}=1, z_{0}>0$. From part $\left.\mathbf{b}\right)$, the condition for unity magnification is

$$
\frac{m_{1}}{\left(\bar{z}+z_{0}\right)^{2}}+\frac{m_{2}}{\left(\bar{z}-z_{0}\right)^{2}}=0
$$


This simplifies into a quadratic equation

$$
\bar{z}^{2}+2\left(m_{1}-m_{2}\right) z_{0} \bar{z}+z_{0}^{2}=0 .
$$

Clearly we have two solutions

$$
\bar{z}_{+,-}=z_{0}\left(\left(m_{2}-m_{1}\right) \pm i \sqrt{1-\left(m_{1}-m_{2}\right)^{2}}\right) .
$$

These two are, as expected, symmetric with respect to the $x$-axis. d) $2(N-1)$. 